\title{
ESTUDO E VALIDAÇÃO DE UMA PROGRESSÃO DE APRENDIZAGEM EM CINÉTICA QUÍMICA DE ESTUDANTES DE NÍVEL SUPERIOR
}

\author{
Luciana G. Monteiro ${ }^{a}$, Antonio C. O. Guerrab e Joaquim F. M. Silva ${ }^{\mathrm{a}, *,(1)}$ \\ aDepartamento de Química Orgânica, Instituto de Química, Universidade Federal do Rio de Janeiro, 21941-909 Rio de Janeiro \\ - RJ, Brasil \\ bDepartamento de Química Inorgânica, Instituto de Química, Universidade Federal do Rio de Janeiro, 21941-909 Rio de Janeiro \\ - RJ, Brasil
}

Recebido em 30/07/2020; aceito em 02/09/2020; publicado na web em 30/09/2020

\begin{abstract}
STUDY AND VALIDATION OF A LEARNING PROGRESSION ON CHEMICAL KINETICS OF HIGHER EDUCATION STUDENTS. This work aims to study the learning progression of students from two federal universities in Rio de Janeiro within the scope of Chemical Kinetics. For the elaboration of the research questionnaire, 46 professors from eleven Brazilian universities were consulted and from the collected data, a questionnaire was elaborated and applied to 452 students of Pharmacy, Chemistry and Chemical Engineering courses at Universidade Federal Fluminense and Universidade Federal do Rio de Janeiro. For the analysis of the questionnaires, Rasch modelling was used, which made it possible to elaborate the learning progression of these students.
\end{abstract}

Keywords: learning progressions; chemical kinetics; higher education; Rasch modelling; chemistry education.

\section{INTRODUÇÃO}

As progressões de aprendizagem (Learning Progressions) são modelos de como a aprendizagem dos estudantes varia ao longo de um determinado período de tempo e que, por isso, podem fornecer informações úteis para a prática docente e para orientar reformas curriculares, ${ }^{1}$ sendo que a primeira aplicação deste modelo foi realizada nos Estados Unidos. ${ }^{2}$ As Progressões de Aprendizagem (PA) podem ser determinadas a partir de indicadores pré-definidos que visam avaliar as efetivas alterações no conhecimento adquirido pelos estudantes em um dado período. ${ }^{3}$ Em outras palavras, no desenvolvimento de uma PA, parte-se de uma hipótese inicial, baseada em pressupostos concretos, tais como estudos anteriores, análise do currículo vigente ou nos demais fatores que influenciam de alguma forma nos processos de ensino e aprendizagem. Nesse contexto, a hipótese inicial poderá ser refutada ou reformulada se o desempenho do sujeito em estudo divergir dos indicadores propostos inicialmente. ${ }^{4}$

Uma questão fundamental nas Progressões de Aprendizagem é que elas devem possuir um ponto de referência inferior e um ponto de referência superior. $\mathrm{O}$ primeiro corresponde ao conhecimento prévio do sujeito em estudo, enquanto o último é definido pelas habilidades que se espera que alcance em relação a um determinado conceito em um período específico. ${ }^{5}$ Entre os limites inferior e superior, existem diversos níveis que podem ser descritos por ideias simplificadas, embasadas cientificamente, ou pela descrição da evolução do entendimento dos estudantes com relação ao conceito ou conhecimento abordado. ${ }^{6}$ Tal evolução revela-se pela mudança na argumentação do sujeito, que também indicará quando ele alcançou o limite superior da progressão. Trata-se, no caso da Ciência, da apropriação e uso dos argumentos científicos em substituição aos argumentos do senso comum. Um exemplo disso é quando o educando consegue trocar a expressão "o calor secou a água" por "ocorreu ebulição". ${ }^{7}$ No trabalho de Schwarz e colaboradores, ${ }^{8}$ os níveis de progressão de aprendizagem foram descritos de acordo com a capacidade dos estudantes de criarem modelos científicos.

\footnotetext{
*e-mail: joaquim@iq.ufrj.br
}

Os níveis de progressão de aprendizagem não são necessariamente lineares ou unidirecionais, podendo haver a passagem de um nível complexo para um nível considerado mais simples a fim de facilitar o entendimento do conceito-alvo. O importante é que todo caminho traçado para a progressão de aprendizagem possa ser produtivo para os estudantes. ${ }^{9}$ Entretanto, as PA não desconsideram as particularidades de cada sujeito - não considera que todos os estudantes aprenderão de maneira única e linear - consistindo em uma sequência idealizada, cujos níveis direcionam o aprimoramento no aprendizado do conhecimento científico, respeitando as habilidades individuais. ${ }^{10}$

Nesse ponto, devemos ressaltar que toda progressão de aprendizagem é considerada hipotética até que seja validada. Essa validação pode se dar por meio empírico - através de rodadas de testes com milhares de alunos - ou estatisticamente. No caso do presente trabalho, utilizamos a modelagem Rasch com auxílio do software Jmetrik. A validação através do modelo de Rasch fornece dados que indicam se o questionário é unidimensional, válido e confiável. ${ }^{1}$

Diferente das abordagens educacionais tradicionais que tratam os conceitos da Química de forma isolada, nas Progressões de Aprendizagem há a interligação das ideias por meio do estudo de como o pensamento químico dos discentes evolui gradativamente. ${ }^{6}$ Além disso, nas PA o ordenamento conceitual de aprendizagem dos estudantes é mais importante que o ordenamento apresentado nos currículos. Com a determinação de quais conceitos os estudantes aprendem com mais facilidade, propõe-se uma reorganização curricular que atenda à progressão de aprendizagem observada. ${ }^{3,11}$

$\mathrm{Na}$ literatura há diversos trabalhos sobre Progressões de Aprendizagem em diferentes áreas do conhecimento. Em Química, especificamente, encontramos trabalhos sobre Termoquímica, ${ }^{4}$ Matéria, ${ }^{12}$ Estruturas Moleculares e suas propriedades, ${ }^{13}$ Substâncias,,${ }^{14}$ Ciclo do Carbono, ${ }^{5}$ Interações químicas entre a água e outras substâncias, ${ }^{2}$ e uma extensa investigação sobre todas as habilidades adquiridas em química desde o ensino fundamental até o fim da graduação. ${ }^{6}$

A finalidade deste estudo é investigar o quanto os estudantes dos cursos de Farmácia, Química e Engenharia Química progridem em termos de habilidades no tocante ao conteúdo de Cinética Química. Escolhemos esse tema, por um lado, porque ele possibilita 
aos docentes a sua correlação com diversos exemplos práticos (como a conservação dos alimentos sob refrigeração e o uso dos combustíveis de foguetes, que são projetados para liberar energia vigorosamente). Por outro lado, porque encontramos na literatura relatos apontando as dificuldades que os estudantes apresentam para utilizar corretamente os conceitos pertinentes. Por isso, diversas concepções alternativas são apresentadas pelos estudantes, dentre as quais, citamos: ${ }^{15}$

- O equívoco relacionado aos conceitos de energia de ativação e entalpia de reação - alguns estudantes acreditam que não há energia de ativação em reações exotérmicas.

- A energia de ativação também é confundida com a energia cinética dos reagentes - os estudantes creem que sempre haverá aumento na velocidade da reação se houver aumento da concentração dos reagentes.

- Os estudantes ainda apresentam dificuldades para esboçar e interpretar gráficos, que são amplamente utilizados nas discussões em Cinética Química.

Nesse contexto, consideramos o estudo da progressão de aprendizagem uma ferramenta interessante para diagnosticar os pontos da Cinética Química onde os estudantes apresentam maior dificuldade. Tal diagnóstico permite instrumentalizar os docentes com dados e informações que os auxiliem na identificação dos meios mais adequados para minimizar as dificuldades de aprendizagem daquele conteúdo. As Progressões de Aprendizagem (PA) visam tornar os processos de ensino e aprendizagem mais efetivos, por meio de uma nova orientação pedagógica, pela adequação dos recursos instrucionais e pela revisão dos instrumentos de avaliação utilizados pelo professor. $^{6}$

O presente trabalho tem por objetivo desenvolver e validar um instrumento de avaliação da progressão de aprendizagem de estudantes de graduação com relação a conceitos específicos da Cinética Química.

\section{PARTE EXPERIMENTAL}

\section{Elaboração do modelo de progressão de aprendizagem a partir das respostas dos especialistas}

A primeira etapa do trabalho foi baseada na pesquisa de Johnson e Tymms, ${ }^{14}$ na qual professores de Química foram consultados com a finalidade de auxiliar no desenvolvimento dos itens de um questionário para os discentes, e no trabalho de Mohan, Chen e Anderson, ${ }^{5}$ em que foi desenvolvida uma progressão de aprendizagem hipotética.

Para essa fase, elaboramos um questionário para ser respondido por professores de Química de diversas universidades brasileiras, que serão chamados de especialistas. O objetivo era construir um primeiro modelo de progressão de aprendizagem sobre alguns conceitos relacionados à Cinética química, um modelo hipotético baseado nas expectativas desses especialistas em relação a como se dá, ou se deveria dar, essa progressão. Na construção desse questionário, levamos em consideração as Orientações Educacionais Complementares aos Parâmetros Curriculares Nacionais ( $\mathrm{PCN}+$ ). $\mathrm{O} \mathrm{PCN}+$ sugere mudanças que proporcionem aos alunos novas habilidades e competências, ao afirmar que:

A proposta apresentada para o ensino de Química nos PCNEM se contrapõe à velha ênfase na memorização de informações, nomes, fórmulas e conhecimentos como fragmentos desligados da realidade dos alunos. Ao contrário disso, pretende que o aluno reconheça e compreenda, de forma integrada e significativa, as transformações químicas que ocorrem nos processos naturais e tecnológicos em diferentes contextos, encontrados na atmosfera, hidrosfera, litosfera e biosfera, e suas relações com os sistemas produtivo, industrial e agrícola. (p. 84) ${ }^{16}$

Dentre as habilidades citadas no PCN+, estão:

- saber interpretar equações químicas, gráficos e diagramas;

- interpretar informações relacionadas à química ambiental em notícias e artigos de jornais, revistas e televisão, sobre agrotóxicos;

- descrever fenômenos utilizando linguagem científica;

- ter a capacidade de posicionar-se de maneira crítica diante de questões científicas e tecnológicas.

Partindo de tais critérios, elaboramos três questões que permitissem investigar a percepção dos especialistas da área sobre a capacidade dos alunos em desenvolverem certas habilidades e competências relacionadas à Cinética Química em três diferentes momentos de sua formação: grupo 1 - no final do Ensino Médio (i.e., ingressantes no Ensino Superior); grupo 2 - no final da disciplina de Química Geral; e grupo 3 - no final do curso de graduação. Optamos por trabalhar os conceitos de Cinética Química de forma aplicada, usando a Química Ambiental como tema de contextualização.

Assim, em cada questão havia a descrição de quatro diferentes habilidades, a partir das quais os especialistas deveriam indicar qual(is) grupo(s) de alunos eles esperariam que tivessem domínio das mesmas. Entre as habilidades investigadas estavam: reconhecer o catalisador e o intermediário de reação num mecanismo simples; escrever as leis de velocidade corretamente; apresentar uma solução para um problema relacionado à conservação da camada de ozônio, com base no conhecimento de cinética química; compreender a função de um catalisador automotivo; e identificar como a luz influencia em uma reação do smog fotoquímico.

O questionário para os especialistas foi criado e disponibilizado em uma plataforma virtual e encaminhado para 758 professores de 14 Instituições de Ensino Superior (IES) brasileiras (UFF, UFRJ, UFRRJ, USP, UNICAMP, UFScar, UFMG, UFRGS, UFSC, UFBA, UFPE, UFG e UFAM). Obtivemos 46 respostas, que foram tratadas considerando um intervalo de confiança de $95 \%$ com margem de erro de $14 \%$.

\section{Investigação da progressão de aprendizagem dos estudantes}

A partir do tratamento dos dados obtidos com a participação dos especialistas, tivemos a noção de quais habilidades dos estudantes deveríamos investigar, bem como quais deveriam ser consideradas mais ou menos complexas para eles. Formulamos, com isso, nossa progressão hipotética e elaboramos um novo questionário para ser respondido pelos alunos dos cursos de Farmácia, Química e Engenharia Química de duas universidades federais brasileiras.

O Quadro 1 apresenta uma breve descrição do questionário utilizado nesse trabalho para verificar a progressão de aprendizagem dos estudantes. Esse questionário continha 12 itens, cujos conceitos (ou domínios) investigativos estavam relacionados aos temas smog fotoquímico, preservação da camada de ozônio e catalisadores automotivos. Na sua estruturação utilizamos questões abertas, de múltipla escolha simples ou de múltipla escolha ordenada, com o objetivo de validar a progressão hipotética elaborada a partir das concepções dos especialistas. No questionário também havia uma questão acerca da origem escolar dos estudantes, cuja finalidade era avaliar se a origem escolar influenciava no desempenho. 
Quadro 1. Descrição dos itens do questionário para verificação da progressão de aprendizagem dos estudantes

\begin{tabular}{|l|l|l|}
\hline Número do item & Formato da Questão & Domínio investigado. \\
\hline 1 & Múltipla escolha ordenada & Identificação de conceitos relacionados à preservação da camada de ozônio \\
\hline 2 & Múltipla escolha & Identificação da expressão correta para a lei de velocidade. \\
\hline 3 & Múltipla escolha & Reconhecimento do catalisador num mecanismo simples. \\
\hline 5 & Questão aberta & $\begin{array}{l}\text { Habilidade de propor medidas para preservar a camada de ozônio, com base nas reações } \\
\text { apresentadas. }\end{array}$ \\
\hline 6 & Múltipla escolha & Reconhecimento do catalisador em um mecanismo complexo \\
\hline 7 & Item dicotômico (duas opç̃̃es de resposta) & Compreensão do conceito de ordem de reação. \\
\hline 8 & Múltipla escolha & $\begin{array}{l}\text { Compreensão em relação ao funcionamento de um catalisador automotivo e interpretação } \\
\text { do mecanismo complexo. }\end{array}$ \\
\hline 9 & Questão aberta & $\begin{array}{l}\text { Escrita da reação global a partir do reconhecimento dos intermediários de reação e do } \\
\text { catalisador. }\end{array}$ \\
\hline 10 & Questão aberta & Compreensão da energia de ativação fornecida pela luz no Smog fotoquímico. \\
\hline 11 & Questão aberta & Descrever experimento no qual a luz influenciasse na ocorrência da reação. \\
\hline 12 & Questão aberta & Habilidade de cálculo acerca das leis de velocidade. \\
\hline & Múltipla Escolha & $\begin{array}{l}\text { Análise e identificação do gráfico mais adequado para o consumo de um reagente na } \\
\text { reação apresentada. }\end{array}$ \\
\hline
\end{tabular}

\section{Construção de modelo de progressão de aprendizagem baseado nas respostas dos estudantes}

Para a análise das respostas dos estudantes aos itens descritos no Quadro 1 e a validação estatística do questionário utilizamos o método Rasch com auxílio do software JMetrik. Segundo Meyer e Hailey, ${ }^{17} \mathrm{o}$ Jmetrik é considerado um software bastante eficiente para modelagem de Rasch. O modelo de Rasch permite a comparação entre estudantes de diferentes níveis e também permite comparar os itens propostos no questionário utilizado nesse trabalho. ${ }^{18}$ Os valores associados à capacidade de um estudante em responder a uma dada questão e o nível de dificuldade dessa questão apresentam a mesma unidade no modelo de Rasch e é a diferença entre esses valores que determina a probabilidade de acerto do estudante. ${ }^{14}$ Uma característica desse modelo é a unidimensionalidade e a independência local. ${ }^{19,20}$

\section{RESULTADOS E DISCUSSÃO}

A etapa de elaboração da progressão de aprendizagem hipotética contou com a participação de 46 docentes de 14 IES brasileiras. Esses especialistas expuseram suas opiniões quanto às habilidades de alunos ingressantes (grupo 1 - considerados de menor nível de instrução), concluintes de química geral (grupo 2 - considerados de nível de instrução intermediário) e formandos (grupo 3 - considerados de maior nível de instrução) acerca de três temas específicos da Cinética Química.
No primeiro tema, Preservação da Camada de Ozônio, as seguintes reações químicas foram apresentadas aos professores: ${ }^{21}$

$$
\begin{aligned}
& \mathrm{NO}(\mathrm{g})+\mathrm{O}_{3}(\mathrm{~g}) \rightarrow \mathrm{NO}_{2}(\mathrm{~g})+\mathrm{O}_{2}(\mathrm{~g}) \\
& \mathrm{NO}_{2}(\mathrm{~g})+\mathrm{O}(\mathrm{g}) \rightarrow \mathrm{NO}(\mathrm{g})+\mathrm{O}_{2}(\mathrm{~g})
\end{aligned}
$$

Os domínios investigados acerca desse tema estão dispostos no Quadro 2.

$\mathrm{Na}$ investigação a respeito do uso dos catalisadores automotivos, foi apresentado o seguinte mecanismo de reação aos especialistas, conhecido como mecanismo de Roginskii:22

$$
\begin{gathered}
\mathrm{O}_{2}(\mathrm{~g})+\mathrm{MnO}_{2}(\mathrm{~s}) \leftrightarrows \mathrm{MnO}_{2} \cdot \mathrm{O}_{2}(\mathrm{~s}) \\
\mathrm{MnO}_{2}(\mathrm{~s})+\mathrm{CO}(\mathrm{g}) \leftrightarrows \mathrm{MnO}_{2} \cdot \mathrm{CO}(\mathrm{s}) \\
\mathrm{MnO}_{2} \cdot \mathrm{CO}(\mathrm{s})+\mathrm{MnO}_{2} \cdot \mathrm{O}_{2}(\mathrm{~s}) \leftrightarrows \mathrm{MnO}_{2} \cdot \mathrm{O}(\mathrm{s})+\mathrm{CO}_{2}(\mathrm{~g}) \\
\mathrm{MnO}_{2} \cdot \mathrm{O}(\mathrm{s})+\mathrm{CO}(\mathrm{g}) \leftrightarrows \mathrm{MnO}_{2}(\mathrm{~s})+\mathrm{CO}_{2}(\mathrm{~g}) \\
\mathrm{MnO}_{2} \cdot \mathrm{CO}(\mathrm{s}) \rightarrow \mathrm{MnO}^{(\mathrm{s})}+\mathrm{CO}_{2}(\mathrm{~g}) \\
2 \mathrm{MnO}(\mathrm{s})+\mathrm{MnO}_{2} \cdot \mathrm{O}_{2}(\mathrm{~s}) \rightarrow 3 \mathrm{MnO}_{2}(\mathrm{~s})
\end{gathered}
$$

\begin{tabular}{|c|c|c|c|}
\hline Domínio investigado & Atribuído ao grupo1 & Atribuído ao grupo 2 & Atribuído ao grupo 3 \\
\hline $\begin{array}{l}\text { Afirmar que a redução da emissão dos óxidos de nitrogênio seria importante } \\
\text { para a preservação da camada de ozônio }\end{array}$ & $48 \%$ & $69 \%$ & $72 \%$ \\
\hline $\begin{array}{l}\text { Escreveria a lei de velocidade de forma correta, caso fossem fornecidos os } \\
\text { dados necessários junto ao mecanismo apresentado. }\end{array}$ & $2 \%$ & $41 \%$ & $87 \%$ \\
\hline $\begin{array}{l}\text { Reconhecer o monóxido de nitrogênio (NO) como um catalisador e o dióxido } \\
\text { de nitrogênio }\left(\mathrm{NO}_{2}\right) \text { como um intermediário. }\end{array}$ & $4 \%$ & $52 \%$ & $80 \%$ \\
\hline $\begin{array}{l}\text { Escrever um texto com argumentos científicos adequados, propondo estraté- } \\
\text { gias para a preservacão da camada de ozônio. }\end{array}$ & $0 \%$ & $13 \%$ & $82 \%$ \\
\hline
\end{tabular}

Com base nesse mecanismo, os especialistas foram indagados sobre as habilidades dispostas no Quadro 3.

Oúltimo tema abordado no questionário dos especialistas foi o Smog Fotoquímico. Nesse tema, o seguinte mecanismo de reação foi exposto: ${ }^{23}$

$$
\begin{gathered}
\mathrm{NO}_{2}(\mathrm{~g})+\mathrm{h} v \rightarrow \mathrm{NO}(\mathrm{g})+\mathrm{O}(\mathrm{g}) \\
\mathrm{O}(\mathrm{g})+\mathrm{O}_{2}(\mathrm{~g}) \rightarrow \mathrm{O}_{3}(\mathrm{~g})
\end{gathered}
$$

Quadro 2. Expectativa dos docentes com relação aos estudantes na investigação sobre o domínio de habilidades em cinética química aplicada à preservação da camada de ozônio 
Quadro 3. Expectativa dos docentes com relação aos estudantes na investigação sobre o domínio de habilidades e em cinética química aplicada ao uso de catalisadores automotivos

\begin{tabular}{|l|c|c|c|}
\hline Domínio investigado & Atribuído ao grupo 1 & Atribuído ao grupo 2 & Atribuído ao grupo 3 \\
\hline $\begin{array}{l}\text { Ser capaz de admitir que a conversão de monóxido de carbono (CO) em } \\
\text { dióxido de carbono }\left(\mathrm{CO}_{2}\right) \text { não ocorreria sem a presença do catalisador. }\end{array}$ & $9 \%$ & $52 \%$ & $74 \%$ \\
\hline Escrever a reação química global para o mecanismo apresentado. & $9 \%$ & $54 \%$ & $80 \%$ \\
\hline $\begin{array}{l}\text { Afirmar que a ordem de reação não apresentará valores absolutos iguais aos } \\
\text { coeficientes da reação global. }\end{array}$ & $4 \%$ & $37 \%$ \\
\hline $\begin{array}{l}\text { Produzir um vídeo informativo sobre problemas ambientais que seriam mi- } \\
\text { nimizados com o uso de catalisadores e com o auxílio dos demais conceitos } \\
\text { de cinética química. }\end{array}$ & $9 \%$ & $78 \%$ \\
\hline
\end{tabular}

Com base nessas informações, os especialistas foram questionados sobre as habilidades dispostas no Quadro 4.

A partir das respostas dos especialistas, elaboramos um novo questionário a ser utilizado como instrumento da avaliação da progressão de aprendizagem do público-alvo desse trabalho. $\mathrm{O}$ instrumento avaliativo continha doze itens e foi aplicado a 452 estudantes, sendo 202 da UFF e 250 da UFRJ. Dentre os participantes da pesquisa 83 eram do curso de Engenharia Química, 171 do curso de Farmácia, 136 eram do curso de Química (Bacharelado, Química industrial e Química com atribuições tecnológicas) e 62 do curso de Licenciatura em Química.

$O$ percentual de acerto para cada grupo de estudante encontra-se descrito no Quadro 5.
Após a observação do percentual de acertos de cada questão pelos três diferentes grupos, realizamos a modelagem de Rasch com auxílio do software JMetric que revelou um índice de confiabilidade dos itens igual a 0,99 , indicando que o instrumento proposto para a avaliação da progressão de aprendizagem é confiável. Uma das características interessantes do modelo de Rasch é que a habilidade dos respondentes e a dificuldade do item são apresentados em valores de logit. ${ }^{24} \mathrm{~A}$ unidade logit possui esse nome por ser uma probabilidade logarítmica. A modelagem de Rasch também fornece os valores das medidas de ajuste chamadas infit e outfit. Um bom ajuste para o modelo Rasch deve ter os valores de infit e outfit situados entre 0,5 e 1,5, considerando-se que um item dentro dessa faixa é válido. ${ }^{25} \mathrm{O}$ Quadro 6 apresenta os valores de ajuste (infit e outfit) e a dificuldade estimada para cada item.

Quadro 4. Expectativa dos docentes com relação aos estudantes na investigação sobre o domínio de habilidades em cinética química aplicada ao Smog fotoquímico

\begin{tabular}{|c|c|c|c|}
\hline Domínio investigado & Atribuído ao grupo 1 & Atribuído ao grupo 2 & Atribuído ao grupo 3 \\
\hline $\begin{array}{l}\text { Presumir que a energia de ativação da formação do oxigênio atômico }(\mathrm{O}(\mathrm{g})) \\
\text { é alcançada através da radiação eletromagnética (luz) proveniente do Sol. }\end{array}$ & $30 \%$ & $52 \%$ & $83 \%$ \\
\hline $\begin{array}{l}\text { Demonstrar, através de cálculos, que o aumento na concentração de dióxido } \\
\text { de nitrogênio }\left(\mathrm{NO}_{2}\right) \text { favorece a formação do ozônio }\left(\mathrm{O}_{3}\right) \text {. }\end{array}$ & $6 \%$ & $52 \%$ & $85 \%$ \\
\hline Esboçar um gráfico da concentração do ozônio $\left(\mathrm{O}_{3}\right)$ versus o tempo. & $2 \%$ & $17 \%$ & $89 \%$ \\
\hline $\begin{array}{l}\text { Propor um experimento para demonstrar a influência da luz na ocorrência } \\
\text { de uma reação química. }\end{array}$ & $2 \%$ & $20 \%$ & $67 \%$ \\
\hline
\end{tabular}

Quadro 5. Percentual de acerto de cada grupo de estudante em cada item do questionário

\begin{tabular}{|c|c|c|c|}
\hline \multirow{2}{*}{ Domínio investigado } & \multicolumn{3}{|c|}{ Execução correta } \\
\hline & Grupo 1 & Grupo 2 & Grupo 3 \\
\hline $\begin{array}{l}\text { Identificou os conceitos relacionados à preservação da camada de ozônio } \\
\text { com base no mecanismo da quebra do ozônio catalisada por óxido nítrico. }\end{array}$ & $88 \%$ & $89 \%$ & $99 \%$ \\
\hline Identificou a expressão correta para a lei de velocidade. & $49 \%$ & $39 \%$ & $79 \%$ \\
\hline Reconheceu o catalisador num mecanismo simples. & $33 \%$ & $33 \%$ & $72 \%$ \\
\hline $\begin{array}{l}\text { Propôs medidas para preservar a camada de ozônio, com base nas reações } \\
\text { apresentadas. }\end{array}$ & $19 \%$ & $20 \%$ & $57 \%$ \\
\hline Reconheceu o catalisador em um mecanismo complexo. & $41 \%$ & $46 \%$ & $77 \%$ \\
\hline Compreendeu o conceito de ordem de reação. & $34 \%$ & $37 \%$ & $59 \%$ \\
\hline $\begin{array}{l}\text { Compreendeu o funcionamento de um catalisador automotivo e interpretou } \\
\text { o mecanismo complexo. }\end{array}$ & $11 \%$ & $13 \%$ & $79 \%$ \\
\hline $\begin{array}{l}\text { Escreveu a reação global a partir do reconhecimento dos intermediários de } \\
\text { reação e do catalisador. }\end{array}$ & $2 \%$ & $0 \%$ & $19 \%$ \\
\hline Compreendeu a energia de ativação fornecida pela luz no Smog fotoquímico. & $10 \%$ & $10 \%$ & $31 \%$ \\
\hline Descreveu um experimento no qual a luz influenciasse na ocorrência da reação. & $30 \%$ & $21 \%$ & $39 \%$ \\
\hline Descreveu as leis de velocidade. & $6 \%$ & $3 \%$ & $9 \%$ \\
\hline $\begin{array}{l}\text { Analisou e identificou o gráfico mais adequado para o consumo de um reagente } \\
\text { na reação apresentada. }\end{array}$ & $44 \%$ & $43 \%$ & $45 \%$ \\
\hline
\end{tabular}


Quadro 6. Valores das estatísticas de ajuste (infit e outfit) e da dificuldade (em logit) obtidos através da modelo de Rasch

\begin{tabular}{|l|c|c|c|c|c|c|}
\hline Item & $\mathbf{1}$ & $\mathbf{2}$ & $\mathbf{3}$ & $\mathbf{4}$ & $\mathbf{5}$ & $\mathbf{6}$ \\
\hline Infit & 0,39 & 1,31 & 0,50 & 0,51 & 1,19 & 1,42 \\
\hline Outfit & 0,59 & 1,21 & 0,63 & 0,59 & 1,07 & 1,35 \\
\hline Dificuldade & $-1,24$ & $-1,33$ & $-0,03$ & 0,40 & $-1,34$ & $-0,97$ \\
\hline Item & $\mathbf{7}$ & $\mathbf{8}$ & $\mathbf{9}$ & $\mathbf{1 0}$ & $\mathbf{1 1}$ & 0,79 \\
\hline Infit & 0,84 & 1,41 & 0,97 & 0,64 & 0,75 & 1,60 \\
\hline Outfit & 1,06 & 0,70 & 0,78 & 0,74 & & 1,90 \\
\hline Dificuldade & 1,06 & 1,51 & 0,78 & 0,32 & $-1,07$ \\
\hline
\end{tabular}

Os valores de dificuldade observados no Quadro 6 nos sugerem que a questão mais difícil foi o item que requeria habilidade de cálculo (item 11, 1,90). O segundo item mais difícil, de acordo com a análise, foi o que solicitava ao estudante que escrevesse a reação global a partir do reconhecimento dos intermediários de reação e do catalisador (item $8,1,51$ ). Por outro lado, eles tiveram grande facilidade para: escrever a lei de velocidade corretamente (item 3, $-0,03$ ), reconhecer o catalisador num mecanismo complexo (item 5 , -1,34) e reconhecer, não apenas o catalisador, mas o intermediário de um mecanismo menos complexo (item 2, -1,33).

Os valores obtidos para os termos infit e outfit são referentes ao ajuste ao modelo de Rasch e comprovaram a unidimensionalidade da amostra de estudantes e a validade do instrumento de avaliação - visto que, com exceção apenas do primeiro e do último, todos os itens do questionário apresentaram valores de infit e outfit dentro do intervalo válido (entre 0,5 e 1,5).

Os valores de dificuldade permitiram organizar a progressão de aprendizagem dos alunos da seguinte forma:

1. As tarefas que os estudantes teriam maior facilidade em realizar (intervalo de dificuldade: $-1,33$ - -0,33 logit), e que poderiam ser descritas como tarefas do primeiro nível de progressão seriam:

- Diferenciar os coeficientes estequiométricos de uma reação dos expoentes numéricos (ordem de reação) das leis de velocidade.

- Reconhecer o catalisador e o intermediário de uma reação em um mecanismo.

- Identificar a expressão correta para a lei de velocidade.

2. No segundo nível da progressão de aprendizagem (intervalo de dificuldade: 0,32-0,78 logit), estão as seguintes habilidades:

- Compreender o conceito de energia de ativação.

- Utilizar exemplos de situações nas quais a luz influencia na ocorrência das reações.

- Propor medidas para diminuir a degradação da camada de ozônio com base nos conhecimentos de cinética química, mais especificamente, a partir da observação de um mecanismo.

3. No terceiro nível da progressão de aprendizagem (intervalo de dificuldade: 1,06 - 1,90 logit), as habilidades esperadas são:

- Conhecer a função dos catalisadores automotivos.

- Realizar cálculos que envolvam leis de velocidade.

- Escrever a reação global de um mecanismo de reação complexo.

\section{CONCLUSÕES}

Este estudo revelou que há uma considerável diferença de desempenho entre os estudantes ingressantes e os formandos, conforme já havia sido previsto pelos especialistas consultados. Contudo, os estudantes ingressantes tiveram desempenho superior ao esperado na identificação de conceitos de cinética química relacionados à preservação da camada de ozônio, enquanto os formandos tiveram desempenho inferior ao previsto pelos especialistas nos itens que requeriam habilidades de cálculo, análise de gráficos, descrição de um experimento no qual a luz fornecesse a energia de ativação necessária para a ocorrência da reação e escrita da reação global de um mecanismo complexo.

Um ponto bastante surpreendente no estudo dessa progressão de aprendizagem é que os concluintes de química geral obtiveram desempenho inferior ou semelhante aos estudantes ingressantes em grande parte dos itens investigados, com exceção apenas do primeiro item do questionário. Isso evidencia que a progressão de aprendizagem dos estudantes não se dá de forma hierarquizada, como esperado pelos docentes.

Com relação à validação estatística, os valores de infit e outfit obtidos para todos os itens - exceto o primeiro e o décimo segundo item - estavam dentro da faixa aceitável para o modelo de Rasch. Esse modelo também se mostrou satisfatório para ordenarmos os itens com base nos valores de dificuldade em logit e, com isso, compreendermos a progressão de aprendizagem dos estudantes.

Por fim, esperamos que o instrumento desenvolvido neste trabalho possa auxiliar os docentes na percepção dos tópicos de Cinética Química nos quais os estudantes têm mais dificuldade, a fim de que os conceitos sejam apresentados e discutidos em sala segundo a progressão de aprendizagem identificada.

\section{AGRADECIMENTOS}

O presente trabalho foi realizado com apoio da Coordenação de Aperfeiçoamento de Pessoal de Nível Superior - Brasil (CAPES) concessão de bolsa de doutoramento à L. G. Monteiro.

\section{REFERÊNCIAS}

1. Todd, A.; Romine, W. L.; Cook Whitt, K.; Sci. Educ. 2017, 101, 32.

2. Salinas, I.; Covitt, B. A.; Gunckel, K. L.; Educ. Quim. 2013, 24, 391.

3. Talanquer, V.; Educ. Quim. 2013, 24, 362.

4. Chen, F.; Zhang, S.; Guo, Y.; Xin, T.; Research in Science Education 2017, 47, 1357.

5. Mohan, L.; Chen, J.; Anderson, C. W.; J. Res. Sci. Teach. 2009, 46, 675.

6. Sevian, H.; Talanquer, V.; Chem. Educ. Res. Pract. 2014, 15, 10.

7. Alonzo, A. C.; Gotwals, A. W.; Learning progressions in science: Current challenges and future directions, Springer Science \& Business Media: Berlin, 2012.

8. Schwarz, C.; Reiser, B. J.; Davis, E. A.; Kenyon, L.; Achér, A.; Fortus, D.; Schwarz, Y.; Hug, B. E.; Krajcik, J.; J. Res. Sci. Teach. 2009, 46, 632.

9. Stevens, S. Y.; Delgado, C.; Krajcik, J. S.; J. Res. Sci. Teach. 2010, 47, 687.

10. Hadenfeldt, J. C.; Bernholt, S., Liu, X.; Neumann, K.; Parchmann, I.; J. Chem. Educ. 2013, 90, 1602. 
11. Steedle, J. T; Shavelson, R. J.; J. Res. Sci. Teach. 2009, 46, 699.

12. Hadenfeldt, J. C.; Neumann, K.; Bernholt, S.; Liu, X.; Parchmann, I.; J. Res. Sci. Teach. 2016, 53, 683.

13. Cooper, M. M.; Underwood, S. M.; Hilley, C. Z.; Klymkowsky, M. W.; J. Chem. Educ. 2012, 89, 1351.

14. Johnson, P.; Tymms, P.; J. Res. Sci. Teach. 2011, 48, 849.

15. Bain, K.; Towns, M. H.; Chem. Educ. Res. Pract. 2016, 17, 246.

16. Brasil. PCN+ Ensino médio: orientações educacionais complementares aos Parâmetros Curriculares Nacionais-Ciências da Natureza, Matemática e suas Tecnologias. 2002.

17. Meyer, J. P.; Hailey, E.; Journal of Applied Measurement 2012, 13, 248.

18. Park, M; Liu, X.; Sci. Educ. 2016, 100, 483.
19. Guedes, E. de S.; E., Orozco-Vargas, L. C.; Turrini, R. N. T.; De Sousa, R. M. C.; Dos Santos, M. A.; Revista Latino-Americana de Enfermagem 2013, 21, 163.

20. Amantes, A.; Coelho, G. R.; Marinho, R.; Ensaio Pesquisa em Educação em Ciências 2015, 17, 657.

21. Wright, J.; Environmental Chemistry, Routledge: Londres, 2003.

22. Rangel, M. Do C; Carvalho, M. F. A.; Quim. Nova 2003, 26, 265.

23. Chang, R.; Goldsby, K. A.; Química, $11^{\mathrm{a}}$ ed., Porto Alegre: AMGH, 2013.

24. Ludlow, L. H.; Haley, S. M.; Educ. Psychol. Meas. 1995, 55, 967.

25. Bergh, D. Em Rasch Measurement: Applications in Quantitative Educational Research; Khyne, M. S., ed.; Springer: Singapore, 2020, cap. 13. 